\title{
Association between dual task-related decrease in walking speed and real versus imagined Timed Up and Go test performance
}

\author{
Stephanie A. Bridenbaugh • Olivier Beauchet • \\ Cédric Annweiler • Gilles Allali • François Herrmann • \\ Reto W. Kressig
}

Received: 20 September 2011 / Accepted: 4 January 2013/Published online: 17 May 2013

(C) Springer International Publishing Switzerland 2013

\begin{abstract}
Background and aims To examine whether older people with markedly dual task-related decreases in walking speed - a marker of disturbed higher-level gait control and falls - have a larger discrepancy between real and imagined Timed Up and Go (TUG) test times than those with less dual task-related decreases in walking speed.

Methods Based on a prospective cross-sectional study, 193 older adults (mean age $77.4 \pm 5.9$ years; $44.0 \%$ women) referred to and consecutively assessed at a Swiss university clinic for a gait analysis to assess either gait disorders, fall risk or memory disorders were included. For all participants, walking speed was measured using a GAITRite $^{\circledR}$ electronic walkway system during usual walking at self-selected pace and while dual tasking (i.e.,
\end{abstract}

S. A. Bridenbaugh $(\bowtie) \cdot$ R. W. Kressig

Acute Geriatrics Department, Faculty of Medicine, Basel

Mobility Center, University Hospital Basel, University of Basel,

Schanzenstrasse 55, 4031 Basel, Switzerland

e-mail: Stephanie.Bridenbaugh@usb.ch

O. Beauchet · C. Annweiler

Division of Geriatric Medicine, Department of Neuroscience, Angers University Hospital, University Memory Clinic of Angers; UPRES EA 4638, University of Angers, Angers, France

G. Allali

Department of Neurology, Faculty of Medicine, Geneva University Hospitals, University of Geneva, Geneva, Switzerland

F. Herrmann

Department of Rehabilitation and Geriatrics, Faculty of Medicine, Geneva University Hospitals, University of Geneva, Geneva, Switzerland usual walking and simultaneously counting backwards out loud). In addition, real Timed Up and Go (TUGr) and imagined Timed Up and Go (TUGi) (i.e., the time needed to imagine performing the TUGr) times were measured with a stopwatch. Differences between both walking conditions for walking speed (delta of walking speed) and both TUG conditions (delta of TUG time) were calculated. Age, gender, height, total number drugs taken per day, daily use of psychoactive drugs, use of walking aid, history of falls, Mini-Mental State Examination score, near vision and education level were used as covariables in this analysis. Results Participants were categorized into two groups based on being in the lowest tertian (i.e., $<33 \%$ : group A corresponding to participants undisturbed by dual task) or not (i.e., $\geq 33 \%$ : group B corresponding to participants disturbed by dual task) of the delta of walking speed. In both groups, TUGr and TUGi times were similar $(P=.169$ and $P=.839$ ). In both groups, TUGi was faster than TUGr $(P<.001)$. Delta of TUG time was significantly greater in group B compared to group A $(P<.001)$. After adjustment for all covariables, only the delta of walking speed was significantly associated with the delta of TUG time $(P=<.001)$. Stepwise backward regression showed that polypharmacy $(P=.017)$ and delta of walking speed $(P=<.001)$ were associated with an increase in delta of TUG time, whereas an increased MMSE score $(P=.030)$ was associated with a decrease in delta of TUG time.

Conclusion These findings show that a large discrepancy between real and imagined TUG performances is significantly correlated with a decrease in walking speed while dual tasking, and thus may also be a surrogate marker of disturbed higher-level gait control. The quickly and easily performed TUG tests may represent a feasible, practical screening tool for early detection of higher-level gait disorders in older adults. 
Keywords Motor imagery - Walking speed - Dual task · Real Timed Up and Go · Imagined Timed Up and Go . Older adults

\section{Introduction}

Decreased walking speed while dual tasking compared to usual walking as a single task has been frequently reported among older adults and has been interpreted as a marker of disturbed higher-level gait control leading to unsafe gait and falls [1-4]. The real (actual) Timed Up and Go Test (TUGr) evaluates mobility in older adults [5] and has been used extensively in geriatric medicine [6]. Recently, it has been shown that an imagined version of the TUG (TUGi) may be used clinically to assess changes in gait control in older adults [7]. More precisely, it was suggested that a large discrepancy between real and imagined TUG times was a marker of disturbed higher-level control of gait and disturbed global cognitive function [7].

Motor imagery, defined as mentally envisioning an action without actually performing it [8], is widely used to investigate the control of movement [9]. Studies have shown that imagined and real movements recruit similar neural networks and preserve the same spatiotemporal characteristics, suggesting that they have similar motor representations [10, 11]. Differences in performance between real and imagined movement have been shown in older as compared to younger adults $[12,13]$ as well as in patients with movement disorders [14-16], suggesting that such discrepancies may mark abnormal control of movement.

Both the dual task-related decrease in walking speed and the difference in performance between real and imagined movement may be related to abnormal higher-level gait control $[1-4,7]$. We hypothesized that discrepancies between the TUGr and its imagined version (TUGi) could be associated with a dual task-related decrease in walking speed. Our objective was to examine whether older people with markedly dual task-related decreases in walking speed-a marker of disturbed higher-level gait control and falls-had a larger discrepancy between TUGr and TUGi times than those with less dual task-related decreases in walking speed.

\section{Methods}

Participants

The 193 participants (mean age $77.4 \pm 5.9$ years; $44.0 \%$ women) in this prospective cross-sectional study were consecutive patients of the Basel Mobility Center between January and August of 2007. Patients were (a) inpatients at the University Hospital Basel, referred by their attending physician for a gait analysis because of gait disorders and/ or falls; (b) community-dwellers, referred by their family physician for a gait analysis because of gait disorders and/ or falls; (c) older cognitively healthy participants of a longitudinal study at the University Hospital Basel Memory Clinic on cognitive changes associated with aging; or (d) outpatients from the University Hospital Basel Memory Clinic, referred by their family physician because of memory problems. Exclusion criteria were inability to walk $15 \mathrm{~m}$ without assistance (from a person or a walking aid) and severe dementia (i.e., diagnosis of dementia made according to the DSM-IV criteria, with a Mini-Mental State Examination (MMSE) [17] score $<10$ ). None of the participants fulfilled the exclusion criteria. Only data from baseline visits were analyzed. There were no drop-outs. Baseline characteristics are summarized in Table 1.

This study was conducted in accordance with the ethical standards set forth in the Helsinki declaration (1983). The study was approved by the local ethics committee.

\section{Clinical assessment}

A patient history was taken from and a medical examination was conducted on all participants by a physician at the Basel Mobility Center or the Memory Clinic. Only the Memory Clinic outpatients had a cognitive diagnosis based on neuropsychological test results, yet the MMSE was performed on all participants included in this analysis. Cognitive impairment was defined as a MMSE score $<26$ points. Information regarding medications was provided by the referring physician and/or reported by the patient. The use of a walking aid, a history of falls in the year prior to gait analysis and the level of education were also selfreported. Near vision was measured at a distance of $0.5 \mathrm{~m}$ with a Snellen letter test chart. Body height and weight were measured with shoes on, since participants wore their shoes for the TUG tests and the gait analysis.

Timed Up and Go test (TUG), real and imagined

The two TUG tests were successively performed in a nonrandomized order: first the real and then the imagined TUG test. The TUGi is based on a mental chronometry approach, which is a tool in neuroscience used to measure the time course of mental operations (7). When using this approach, the first step is to perform the motor test and then the second step is to imagine the same motor test. Thus, the order cannot be randomized. 
Table 1 Characteristics of participants $(n=193)$ based on differences in walking speed

\begin{tabular}{|c|c|c|c|}
\hline & $\begin{array}{l}\text { Group A } \\
(n=134)\end{array}$ & $\begin{array}{l}\text { Group B } \\
(n=59)\end{array}$ & $P$ value $^{\dagger}$ \\
\hline Age, mean \pm SD (years) & $77.3 \pm 5.7$ & $77.6 \pm 6.5$ & 0.782 \\
\hline Female, $n(\%)$ & $51(38.1)$ & $34(57.6)$ & 0.018 \\
\hline Height, mean \pm SD $(\mathrm{cm})$ & $169.6 \pm 9.4$ & $166.3 \pm 9.3$ & 0.028 \\
\hline $\begin{array}{l}\text { Total number drugs per day, } \\
\text { mean } \pm \text { SD }\end{array}$ & $4.0 \pm 3.3$ & $3.6 \pm 2.6$ & 0.453 \\
\hline $\begin{array}{l}\text { Use of psychoactive drugs } \\
\text { per day }{ }^{\mathrm{a}}, n(\%)\end{array}$ & $28(20.9)$ & $19(32.2)$ & 0.149 \\
\hline Use of walking aid, $n(\%)$ & $17(12.7)$ & $8(13.6)$ & 0.517 \\
\hline History of falls, $n(\%)$ & $69(51.5)$ & $28(47.5)$ & 0.633 \\
\hline \multicolumn{4}{|l|}{ MMSE (points/30) } \\
\hline Mean \pm SD & $27.6 \pm 2.6$ & $25.2 \pm 3.9$ & $<0.001$ \\
\hline$<26, n(\%)$ & $25(18.7)$ & $25(42.4)$ & 0.001 \\
\hline $\begin{array}{l}\text { Near vision }(/ 1) \\
\text { mean } \pm \mathrm{SD}\end{array}$ & $0.5 \pm 0.3$ & $0.6 \pm 0.3$ & 0.333 \\
\hline $\begin{array}{l}\text { Level of education, } \\
\text { mean } \pm \mathrm{SD} \text { (years) }\end{array}$ & $12.6 \pm 3.3$ & $11.5 \pm 3.0$ & 0.047 \\
\hline \multicolumn{4}{|l|}{ Gait speed, mean $\pm \mathrm{SD}(\mathrm{cm} / \mathrm{s})$} \\
\hline Single task: usual walking & $111.6 \pm 28.1$ & $106.0 \pm 26.8$ & 0.196 \\
\hline $\begin{array}{l}\text { Dual task: working } \\
\text { memory task }\end{array}$ & $98.4 \pm 27.6$ & $61.6 \pm 22.2$ & $<0.001$ \\
\hline
\end{tabular}

Participants were categorized into two groups based on being in the lowest tertian (i.e., $<33 \%$ : group A corresponding to individuals with lowest dual task-related gait changes) or not (i.e., $\geq 33 \%$ : group B corresponding to individuals with higher dual task-related gait changes) of delta of walking speed. Delta speed $=[(\mathrm{UW}$ speed $-\mathrm{DT}$ speed $) /$ $($ UW speed + DT speed $) / 2] \times 100$

$S D$ Standard deviation, MMSE Mini-Mental State Examination [17]

$\dagger$ Based on independent or paired samples $t$ test or Chi-square, as appropriate

$P$-values marked in bold are significant $(P<0.05)$

${ }^{a}$ Psychoactive drugs included anxiolytics, neuroleptics, antidepressants, antiepileptics and antidementia drugs

\section{TUG real (TUGr)}

Testing procedures have been previously described [5, 7]. In brief, participants sat in a chair (with armrests, seat $46 \mathrm{~cm}$ from the ground) and received the standardized verbal instructions from the test administrator to (at the word "go") stand up, walk at their normal pace around a small traffic cone (height $23 \mathrm{~cm}$, diameter of base $18.5 \mathrm{~cm}$ ) $3 \mathrm{~m}$ away, return to the chair and sit down again [5]. If the verbal instructions were not understood, the test administrator provided a visual demonstration by walking the course. If the participant had no questions, the test was performed without a practice trial. Performance was timed to the nearest full second with a stopwatch, which was started at the word "go" ("ready-set-go") and stopped when the participant was seated again. The test was not repeated. Participants were not informed of their test time performance. Participants wore their normal shoes and performed the TUGr without walking aids. Two slight differences to the original test description by Podsiadlo and Richardson are to be noted: the 3-m distance was marked with a small traffic cone rather than a line on the floor, and participants sat with their arms resting either on the chair's arms (as described by Podsiadlo and Richardson) or in their lap, whichever the participant did spontaneously.

\section{TUG imagined (TUGi)}

After the TUGr, the participants remained seated and the TUGi was administered. Participants received standardized verbal instructions to, at the word "go", imagine performing the TUGr and to then say "stop" when, in their imagined performance, they sat back down. Performance was timed to the nearest full second with a stopwatch, which was started at the word "go" ("ready-set-go") and stopped when the participant said "stop".

\section{Gait analysis}

Gait analyses were performed according to the European guidelines for clinical applications of spatio-temporal gait analysis in older adults [18] using the GAITRite ${ }^{\circledR}$ system (GAITRite $^{\circledR}$ Gold, CIR Systems, PA, USA). This system consists of a $972 \mathrm{~cm}$ long electronic walkway with integrated pressure sensors placed every $1.27 \mathrm{~cm}$ over an active electronic surface area of $792 \times 610 \mathrm{~cm}$, giving a total of 29,952 sensors. The scanning frequency was $60 \mathrm{~Hz}$. Data from the mechanically activated sensors were collected by onboard processors and transferred via cable and serial port to a computer and analyzed with the GAITRite $^{\circledR}$ software version 3.8. The walkway was flanked at the beginning and end by $1.25 \mathrm{~m}$ long optically identical yet electronically inactive walkway sections. Acceleration and deceleration phases of gait occurred on these electronically inactive sections, insuring measurement of gait parameters under steady state conditions. Participants wore their normal shoes and performed the gait analysis without walking aids.

Before testing, standardized verbal instructions regarding the test procedure were given by the test administrator, as well as a visual demonstration if the verbal instructions were not understood. No practice walks were performed before testing.

Each participant performed two walks. The first was the single task of walking forward at a self-selected pace (referred to in this text as usual walking and abbreviated UW). The second was the dual task of usual walking while simultaneously performing serial subtractions from 50 by $2 \mathrm{~s}$ out loud (working memory task). No instructions regarding task prioritization were given. Each participant performed each walk once. 
Safety measures

During the TUGr and the gait analysis, participants wore a Posey ${ }^{\circledR}$ safety belt around their waist and were accompanied by a test administrator walking at arm's length allowing easy grasp of the belt should a subject have stumbled.

\section{Outcome measures}

The following outcome measures were recorded: mean $\pm \mathrm{SD}$ of walking speed $(\mathrm{cm} / \mathrm{s})$ during usual walking and during dual tasking, and the mean $\pm \mathrm{SD}$ of TUGr and TUGi times (s). Age (years), gender, height $(\mathrm{cm})$, total number of drugs taken daily, daily use of psychoactive drugs (anxiolytics, neuroleptics, antidepressants, antiepileptics, antidementives), use of a walking aid, history of falls in the year prior to gait analysis, MMSE score, near vision and education level (years) were used as covariates in the data analysis.

\section{Statistics}

The participants' baseline characteristics were summarized using means and standard deviations or frequencies and percentages, as appropriate. The normality of the parameters' distribution was verified with skewness and kurtosis tests before and after applying the usual transformations to normalize non-Gaussian variables. Relative differences between usual walking [19] and dual task (DT)-related walking speeds were calculated as percentages of the mean according to the following formula: delta speed $=[(\mathrm{UW}$ speed - DT speed)/(UW speed + DT speed $) / 2] \times 100$. We used this calculated parameter because the decrease in motor performance between two conditions-i.e., single and dual task for walking speed and real and imagined conditions for TUG times-is strongly related to the level of performance of the reference task, which was in our study, respectively, the usual walking speed and the TUGr. Thus, the unique way to control the level of motor performance is to use a relative difference. Participants were categorized into two groups based on being in the lowest tertian (i.e., $<33 \%$ : group A corresponding to participants undisturbed by dual task) or not (i.e., $\geq 33 \%$ : group $\mathrm{B}$ corresponding to participants disturbed by dual task) of the delta of walking speed (Table 1). In addition, relative differences between TUGr and TUGi times were calculated as percentages of the mean according to the following formula: delta time $=[(\mathrm{TUGr}-\mathrm{TUGi}) /(\mathrm{TUGr}+\mathrm{TUGi}) /$ 2] $\times 100$.

Comparisons between groups were performed using independent or paired samples $t$ test, or Chi-square, as appropriate. Univariate and multiple linear (i.e., fully and stepwise backward models) regression analyses were performed to specify the association between delta of TUG time (dependent variable) and baseline characteristics (independent variables). In multiple linear regression analyses, all baseline characteristics were selected in the models. $P$ values $<0.05$ were considered statistically significant. All statistics were performed using the Stata Statistical Software release 15.0.

\section{Results}

All participants were able to complete the TUGr, the TUGi and all walks of gait analysis. As shown in Table 1, comparisons between the groups showed that participants who were more disturbed by dual task (i.e., group B) were more frequently female $(P=.018)$, had shorter height $(P=.028)$, had a lower MMSE score $(P=.001)$ and a lower education level $(P=.047)$ than those who were undisturbed by dual task (i.e., group A). There were no significant differences between groups for the other characteristics. In both groups, the TUGi times were significantly shorter than the TUGr times $(P<0.001)$. There were no significant differences between the groups for the TUGr $(P=.169)$ and the TUGi $(P=.839)$ times. The delta of TUG time was significantly greater in group B compared to group A $(P<.001)$ (Fig. 1).

Table 2 displays the results from univariate and multiple linear regressions showing the association between the delta of TUG time and the characteristics of participants. It shows that female gender and increased delta of walking speed were associated with an increase in the delta of TUG time $(P=.001$ and $P<0.001)$, whereas an increase in height, MMSE score and education level were associated with a decrease in the delta of TUG time (respectively, $P=.048, P=<.001$ and $P=.038$ ). After adjustment for all covariables, only the increase in delta of walking speed $(P=<.001)$ was significantly associated with the increase in delta of TUG time. Finally, stepwise backward regression showed that an increase in the number of medications taken daily $(P=.017)$ and in the delta of walking speed $(P=<.001)$ were associated with increased delta of TUG time, whereas an increased MMSE score $(P=.030)$ was associated with a decrease in delta of TUG time.

\section{Discussion}

Our findings show that older adults who walked considerably slower while dual tasking compared to single tasking had a greater difference between real and imagined TUG times than those with less dual task-related gait interference. 
Fig. 1 Comparsion between TUGr and TUGi times and the delta of TUG times. Real Timed Up and Go (TUGr), Imagined Timed Up and Go (TUGi), TUG delta time $-[($ TUGr - TUGi $) /$ $(\mathrm{TUGr}+\mathrm{TUGi}) / 2] \times 100$.

$\uparrow$ Participants were categorized in wo groups based on being in the lowes tertial (i.e. less than $33 \%$ : group A) or not (i.e. equal or greater than $33 \%$ : group B) of delta galt speed. Delta speed $=[(\mathrm{UW}$ speed DT speed $) /($ UW speed + DT speed)/2] $\times 100$

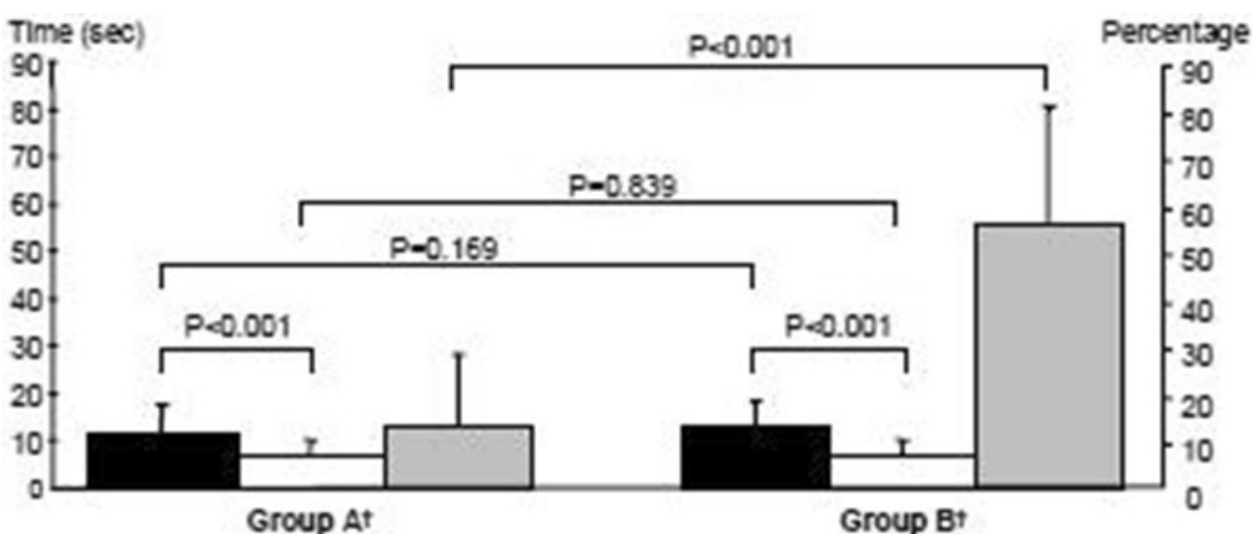

Timad up \&. Go rea time (sec) Timad up \& Go imagined time (sec) Timed up \&. Go desta tme (\%)

Table 2 Univariate and multiple linear regressions examining the association between the delta of TUG time (dependent variable) and participants' characteristics $(n=193)$

\begin{tabular}{|c|c|c|c|c|c|c|c|c|c|}
\hline & \multicolumn{3}{|l|}{ Crude model } & \multicolumn{3}{|c|}{ Fully model } & \multicolumn{3}{|c|}{ Stepwise backward model } \\
\hline & $\begin{array}{l}\text { Unadjusted } \\
\text { effect }^{\mathrm{a}}\end{array}$ & $95 \% \mathrm{CI}$ & $P$ value & $\begin{array}{l}\text { Adjusted } \\
\text { effect }^{\mathrm{a}}\end{array}$ & $95 \% \mathrm{CI}$ & $P$ value & $\begin{array}{l}\text { Adjusted } \\
\text { effect }^{\mathrm{a}}\end{array}$ & $95 \% \mathrm{CI}$ & $P$ value \\
\hline Age & -0.2 & {$[-0.8 ; 0.5]$} & 0.565 & 0.2 & {$[-0.6 ; 0.7]$} & 0.934 & - & - & - \\
\hline Female & 12.7 & {$[5.2 ; 20.2]$} & 0.001 & 6.3 & {$[-5.1 ; 17.8]$} & 0.276 & - & - & - \\
\hline Height & -0.4 & {$[-0.8 ; 0.1]$} & 0.048 & 0.2 & {$[-0.3 ; 0.8]$} & 0.426 & - & - & - \\
\hline $\begin{array}{l}\text { Total number of drugs taken } \\
\text { daily }\end{array}$ & 0.1 & {$[-1.2 ; 1.4]$} & 0.912 & 1.2 & {$[-0.1 ; 2.5]$} & 0.067 & 1.4 & {$[0.3 ; 2.6]$} & 0.017 \\
\hline $\begin{array}{l}\text { Number of psychoactive } \\
\text { drugs taken daily }\end{array}$ & 8.9 & {$[-0.2 ; 17.9]$} & 0.054 & 0.4 & {$[-8.8 ; 9.6]$} & 0.937 & - & - & - \\
\hline Use of walking aid & 0.2 & {$[-11.2 ; 11.6]$} & 0.976 & 5.2 & {$[-6.9 ; 17.3]$} & 0.397 & - & - & - \\
\hline $\begin{array}{l}\text { History of falls in previous } \\
\text { year }\end{array}$ & -4.5 & {$[-12.3 ; 3.4]$} & 0.260 & 0.9 & {$[-6.3 ; 8.0]$} & 0.806 & - & - & - \\
\hline MMSE & -2.6 & {$[-3.8 ;-1.5]$} & $<0.001$ & -1.0 & {$[-2.1 ; 0.0]$} & 0.059 & -1.1 & {$[-2.1 ;-0.1]$} & $\mathbf{0 . 0 3 0}$ \\
\hline Near vision score & 11.0 & {$[-1.4 ; 23.4]$} & 0.081 & 8.3 & {$[-3.6 ; 20.1]$} & 0.169 & - & - & - \\
\hline Level of education & -1.4 & {$[-2.8 ;-0.1]$} & 0.038 & -0.1 & {$[-1.3 ; 1.1]$} & 0.924 & - & - & - \\
\hline Delta speed & 55.4 & {$[46.5 ; 64.3]$} & $<0.001$ & 53.8 & {$[42.9 ; 64.7]$} & $<0.001$ & 55.4 & {$[45.1 ; 65.6]$} & $<0.001$ \\
\hline
\end{tabular}

$N=193$ participants from whom all data were available, intercept value not shown in the table

Delta time $=[(\mathrm{TUGr}-\mathrm{TUGi}) /(\mathrm{TUGr}+\mathrm{TUGi}) / 2] \times 100, \mathrm{TUGr}=$ real TUG time, TUGi $=$ imagined TUG time

Delta speed $=[(\mathrm{UW}-\mathrm{DT}) /(\mathrm{UW}+\mathrm{DT}) / 2] \times 100, \mathrm{UW}=$ usual walking speed, DT $=$ walking speed while dual tasking

CI Confidence interval, MMSE Mini-Mental State Examination [17]

$P$-values marked in bold are significant $(P<0.05)$

${ }^{a}$ Effect estimated from coefficient of regression beta and corresponding to an increase of mean value of TUG delta value

Motor imagery is a well-known technique among athletes to improve functional outcome [20]. It has been used successfully in rehabilitation medicine to improve motor performance, for example in poststroke hemiparesis [15, 21, 22]. Several studies have examined the association between motor imagery and motor performance to investigate the neuro-anatomical correlates and the higher-level control of movement. Many of these studies have focused on relatively simple upper body movements, such as finger tapping, hand or eye movements [23-26]. 
In a comparison of several functional brain imaging studies investigating the motor imagery of different aspects of gait, Malouin et al. [11] summarized those results by stating that imagined and actually performed locomotion activate cortical networks that greatly overlap. Several studies have proposed that dual task-related decreased walking speed is a marker of disturbed higher-level gait control leading to unsafe gait and falls [1-4]. It has been recently proposed that discrepancies between TUGr and TUGi in older adults also represent disturbed higher-level gait control [7]. Thus, it is feasible that disturbances in these cortical networks could be detected both by actual motor and by motor imagery assessments. The results of the present study support this concept.

Mental chronometric studies in healthy adults showed similar times between real and imagined walking and that the time for both covert and overt walking increased with increased task difficulty $[9,11]$. In our study, there were no significant differences in the real TUG times between groups A and B. This means that the TUG delta time difference between the groups was not due to overall worsened mobility in group B (the group with greater dual-task interference on gait speed) but rather due to differences in the imagined TUG times. In both groups A and B, the imagined TUG times were shorter than the real TUG times, meaning that for both groups imagined performance was faster than actual performance.

In our study, stepwise backward regression showed that polypharmacy (i.e., $\geq 4$ drugs taken daily) was associated with increased TUG delta time. It has been reported that polypharmacy in transitionally frail older adults is closely correlated with dual task-related gait changes, such as decreased walking speed [27]. This, however, cannot sufficiently explain the association with increased TUG delta times shown in our results, since there were no significant differences between the TUGr times in our two groups. It may be that polypharmacy affects motor imagery ability in older adults.

Also in our results, stepwise backward regression revealed that an increased MMSE score $(P=.030)$ was associated with a decrease in the delta of TUG time. This corroborates previous results [7]. Although the MMSE is a global measure of cognition, it is not a particularly good measure of executive function. Impaired executive function is often present in cognitive decline and is associated with impaired gait performance under dual-task conditions [13]. Cognitive deficits, particularly executive dysfunction, may, in part, account for the increased delta of TUG time in the group of participants with dual-task impaired gait.

There are some limitations to our study and the interpretation of our results. Since our participants were from different clinical settings, results may not be representative of other older adult populations. We did not stratify our results (deltas of walking speed and of TUG times) according to different age groups or levels of cognition, both of which may influence motor imagery ability [7].

\section{Conclusion}

We show that a dual task-related decrease in walking speed is positively associated with large delta of TUG times (real versus imagined performance times) in older adults. Large discrepancies between real and imagined TUG times may represent disturbed higher-level control of locomotion, which can cause unsafe gait. We propose that these quick and clinically feasible tests, real and imagined TUG tests, could be used in various clinical settings to determine which older patients may have an underlying dual taskrelated gait disturbance and require an in-depth functional gait assessment. Early detection of gait impairment allows for timely and preventive interventions to improve gait before functional mobility declines and/or before a fall occurs.

\section{Conflict of interest None.}

\section{References}

1. Beauchet $\mathrm{O}$ et al (2005) Dual-task-related gait changes in the elderly: does the type of cognitive task matter? J Mot Behav 37(4):259-264

2. Beauchet $\mathrm{O}$ et al (2005) Dual-task-related gait changes in transitionally frail older adults: the type of the walking-associated cognitive task matters. Gerontology 51(1):48-52

3. Sheridan PL et al (2003) Influence of executive function on locomotor function: divided attention increases gait variability in Alzheimer's disease. J Am Geriatr Soc 51(11):1633-1637

4. Kressig RW et al (2008) Gait variability while dual-tasking: fall predictor in older inpatients? Aging Clin Exp Res 20(2):123-130

5. Podsiadlo D, Richardson S (1991) The timed "Up \& Go": a test of basic functional mobility for frail elderly persons. J Am Geriatr Soc 39(2): 142-148

6. Shumway-Cook A, Brauer S, Woollacott M (2000) Predicting the probability for falls in community-dwelling older adults using the Timed Up \& Go Test. Phys Ther 80(9):896-903

7. Beauchet $\mathrm{O}$ et al (2010) Imagined Timed Up \& Go test: a new tool to assess higher-level gait and balance disorders in older adults? J Neurol Sci 294(1-2):102-106

8. Jeannerod M (1994) The representing brain: neural correlates of motor intention and imagery. Behav Brain Sci 17(2):187-245

9. Bakker $M$ et al (2007) Motor imagery of gait: a quantitative approach. Exp Brain Res 179(3):497-504

10. Personnier $\mathrm{P}$ et al (2010) Temporal features of imagined locomotion in normal aging. Neurosci Lett 476(3):146-149

11. Malouin F, Richards CL (2010) Mental practice for relearning locomotor skills. Phys Ther 90(2):240-251

12. Hutchinson $S$ et al (2002) Age-related differences in movement representation. Neuroimage 17(4):1720-1728

13. Mulder T et al (2007) Motor imagery: the relation between age and imagery capacity. Hum Mov Sci 26(2):203-211 
14. Mutsaarts M, Steenbergen B, Bekkering H (2007) Impaired motor imagery in right hemiparetic cerebral palsy. Neuropsychologia 45(4):853-859

15. de Vries S, Mulder T (2007) Motor imagery and stroke rehabilitation: a critical discussion. J Rehabil Med 39(1):5-13

16. Yaguez L et al (1999) Motor learning by imagery is differentially affected in Parkinson's and Huntington's diseases. Behav Brain Res 102(1-2):115-127

17. Folstein MF, Folstein SE, McHugh PR (1975) "Mini-mental state". A practical method for grading the cognitive state of patients for the clinician. J Psychiatr Res 12(3):189-198

18. Kressig RW, Beauchet O (2006) Guidelines for clinical applications of spatio-temporal gait analysis in older adults. Aging Clin Exp Res 18(2):174-176

19. de Laat KF et al (2010) Gait in elderly with cerebral small vessel disease. Stroke 41(8):1652-1658

20. Wei G, Luo J (2010) Sport expert's motor imagery: functional imaging of professional motor skills and simple motor skills. Brain Res 1341:52-62

21. Dunsky A et al (2008) Home-based motor imagery training for gait rehabilitation of people with chronic poststroke hemiparesis. Arch Phys Med Rehabil 89(8):1580-1588
22. Dunsky A et al (2006) Motor imagery practice in gait rehabilitation of chronic post-stroke hemiparesis: four case studies. Int $\mathrm{J}$ Rehabil Res 29(4):351-356

23. Gentili R et al (2010) Motor learning without doing: trial-by-trial improvement in motor performance during mental training. J Neurophysiol 104(2):774-783

24. Ueno $\mathrm{T}$ et al (2010) Comparison between a real sequential finger and imagery movements: an FMRI study revisited. Brain Imaging Behav 4(1):80-85

25. Fleming MK, Stinear CM, Byblow WD (2010) Bilateral parietal cortex function during motor imagery. Exp Brain Res 201(3): 499-508

26. Sauvage $C$ et al (2011) Reevaluating brain networks activated during mental imagery of finger movements using probabilistic Tensorial Independent Component Analysis (TICA). Brain Imaging Behav 5(2):137-148

27. Beauchet $\mathrm{O}$ et al (2005) Relationship between dual-task related gait changes and intrinsic risk factors for falls among transitional frail older adults. Aging Clin Exp Res 17(4):270-275 